\title{
Применение ВЭЖХ совместно с оптическими и термическими методами в комплексном анализе межкомпонентных взаимодействий фармацевтических композиций
}

\author{
Ельцова Н.О. ${ }^{1}$, Будко Е.В. ${ }^{2}$ \\ ${ }^{l}$ ООО «СТЦ», Санкт-Петербург \\ ${ }^{2}$ ФГБОУ ВО Курский государственный медицинский университет МЗ России, Курск
}

Поступила в редакцию 26.01.2019 г.

DOI: https://doi.org/10.17308/sorpchrom.2019.19/786

Разнообразие новых лекарственных препаратов и возрастающие требования применяемого к ним стандарта качества требуют систематического подхода к исследованию возможного взаимодействия компонентов. Комплексное применение оптических, хроматографических и термоаналитических методов в сочетании с воздействием стресс-факторов позволило исследовать процессы и дать характеристику продуктов деструкции. По результатам опыта работы с группой многокомпонентных препаратов анальгетического, противовоспалительного и противопростудного действия сформулирован единый методический подход к процессу изучения стабильности фармацевтических субстанций в составе лекарственных композиций.

Ключевые слова: методы анализа, ИК-спектроскопия, УЭЖХ, калориметрические методы анализа, фармацевтические субстанции, анализ лекарственных препаратов

\section{Application of HPLC with optical and thermal methods in complex analysis of inter-component interactions of pharmaceutical compositions}

\author{
Eltsova N.O., Budko E.V. \\ ${ }^{1}$ LLC STC, St. Petersburg \\ ${ }^{2}$ Kursk state medical University, Kursk
}

\begin{abstract}
The variety of new drugs and the increasing requirements of the GMP (Good Manufacturing Practice for medical Products) standard applied to them require a systematic approach to the study of possible interaction of components. It is extremely important not only to ascertain the compliance of the drug with the established requirements but also to understand the causes of undesirable deviations. Chromatographic, optical, thermal methods of analysis, provided with fairly modern and widespread equipment and mathematical apparatus, are most often used to identify inter-component interaction. The joint use of the methods allows obtaining complex and realistic information about the mixtures, to assess the state of the components during their contact, including as a result of the impact of stress conditions. Based on the results of experience with a group of multicomponent drugs analgesic, anti-inflammatory and anti-cold action formulated a unified methodological approach to the process of studying the stability of pharmaceutical substances in the composition of drug compositions. The algorithm includes the following blocks. The choice of objects (can be limited to the prescription of the drug or expanded by analogs). Detection of deviations in the IR spectra of the mixture and the sum of the spectra of individual ingredients. Evaluation of thermograms and state diagrams. Computational and graphical analysis of the digital data Bank (allows identifying interaction products with
\end{abstract}


greater confidence in the result). HPLC (or it's variant of HPLC) analysis (to confirm the absence of significant amounts of impurities and the quantitative content of active components) at the final stage allows proving the stability of the components. The complex application of optical, chromatographic and thermoanalytical methods in combination with the influence of stress factors and (or) in the conditions of "accelerated aging" made it possible to study the processes and characterize the degradation products more effectively than each of the methods separately. The result of the research obtained data on the stability of 11 pairs of compounds: drotaverine and magnesium, calcium stearate, drotaverine and citric acid, and the GOC drotaverine, drotaverine and croscarmellose sodium, naproxen and magnesium, calcium stearate, and the GOC naproxen, naproxen and pheniramine maleate, pheniramine maleate and the GOC, pheniramine maleate and ascorbic acid, co-grinding and heating which strengthens the processes of inter-pillar interaction.

Keywords: methods of analysis, IR spectroscopy, HPLC, calorimetric methods of analysis, pharmaceutical substances, drug analysis

\section{Введение}

На биологическую и химическую активность фармацевтических ингредиентов оказывает влияние не только химическое состояние вещества, но и физическое полиморфизм, степень дисперсности и агрегатное состояние. В частности, явление полиморфизма распространено среди салицилатов - ацетилсалициловая кислота встречается в шести кристаллических формах, превращения между которыми проходят при истирании, прессовании, гранулировании, дражировании, а активирующим фактором является увлажнение. Полиморфные модификации имеют различную стойкость к деструктивным процессам и изменение поверхностных свойств, от которых зависит биодоступность и стабильность компонентов в лекарственных формах. Вспомогательные вещества так же способны вступать во взаимодействие другом с другом, с активными фармацевтическими ингридиентами и с внешней средой. Между веществами могут устанавливаться различные типы связей - Ван-дер-Ваальсовы, водородные, ковалентные, возможно образование соединений включения, комплексов и т.д. Все это может влиять на стабильность препаратов [1].

Разнообразие новых лекарственных препаратов и возрастающие требования применяемого к ним стандарта GMP (Good Manufacturing Practice for Medicinal Products) требуют систематического подхода к исследованию возможного взаимодействия компонентов. Чрезвычайно актуальна не только констатация соответствия препарата установленным требованиям, но и понимание причин нежелательных отклонений. Взаимодействие может происходить в процессе производства лекарственного препарата под воздействием стресс-факторов - давления и температуры и в процессе хранения. В дальнейшем это сказывается и на эффективность применения лекарственного средства и осложняет технологические и аналитические задачи производства препарата. Для предотвращения взаимодействия предлагаемые технологические походы должны быть основаны на представлениях о структурных изменениях компонентах в процессе производства [2]. В таком случае возможно исключить деструкцию компонентов путем изменения технологических параметров или компонентного состава.

Разнообразие новых лекарственных препаратов, сложность их химического состава требует дальнейшего развития аналитической базы для регистрации изменений, возможных в процессе приготовления и хранения препаратов. В периодической литературе опубликовано немало работ о взаимодействии компонентов и определении продуктов их деструкции. Для выявления межкомпонентного взаимодействия чаще всего используются хроматографические, оптические, термические методы анализа, обеспеченные достаточно современным и широко распространённым оборудованием и математическим аппаратом. Хроматографические методы - ВЭЖХ, ГЖХ с различными способами детектирования (УФ, МС и др.) - широко применяют-

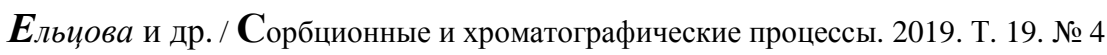


ся в фармацевтическом анализе. Ни один другой физико-химический метод не может сравниться с хроматографией по эффективности при анализе сложных смесей. Этому способствуют достижения в области разработки новых сорбентов, повышение чувствительности и разрешающей способности детекторов. Спектроскопия обеспечивает быстрый неразрушающий анализ и простой путь контроля процессов, при которых наблюдаются спектральные изменения. Оптические методы (ИК-, УФ- и видимой областях спектра) часто используются для изучения процессов деструкции, хотя при подобии спектральных характеристик примесей и основного вещества обнаружение продуктов разложения затруднительно. К спектральным методам принято относить и масс-спектрометрию (МС), а также ЯМР-спектроскопию. Термические методы (ДСК, ТГ) широко используются в анализе стабильности и влажности материалов, компонентного состава органических и неорганических форм, входящих в состав исследуемой смеси [3].

Расхождение между результатами, полученными различными методами в исследованиях взаимодействия и совместимости, встречаются часто. Это связано с различиями изучаемых процессов при различных условиях (температура, среда), необходимых для осуществления метода. Однако совместное использование методов позволяет получать комплексную и реалистичную информацию о смесях, проводить оценку состояния компонентов при их контакте, в том числе в результате воздействия стрессовых условий. В таблице 1 обобщены сведения совместного применения методов при анализе стабильности фармацевтических композиций. Обзор публикаций показывает наибольшую частоту применения пар спектральные - хроматографические (ВЭЖХ), совместное применение нескольких спектральных (ИК, МС ЯМР), или их сочетание с термическими (ДСК и ТГ). Для контроля стабильности и идентификации продуктов разложения лекарственных препаратов особенно перспективно сочетание ВЭЖХ с МС и ЯМР, позволяющее не только разделить, но и идентифицировать неизвестные продукты.

Таблица 1. Возможные сочетания методов при анализе стабильности фармацевтических композиций

\begin{tabular}{|c|c|c|c|c|}
\hline Методы анализа & ВЭЖХ & ТГ & МС & ИК \\
\hline ИК & {$[7]$} & {$[3]$} & {$[4,7]$} & \\
\hline МС & {$[3,5,6,7]$} & {$[8]$} & & \\
\hline ЯМР & {$[5,6]$} & & & {$[4,7]$} \\
\hline
\end{tabular}

На основе знания механизма взаимодействия и деструкции компонентов можно сделать важные шаги в управлении качеством лекарственного препарата. Например, для предотвращения деструкции сокращают время смешивания и снижают температуру сушки, исключают взаимодействие компонентов путем изменения технологических параметров или компонентного состава. Представление о структурных изменениях компонентов в смеси позволяет изменять скорость растворения в средах организма и потенциально влиять на биодоступность.

Целью работы было разработать методический подход к процессу изучения стабильности фармацевтических субстанций в составе лекарственных композиций в твердой фазе на основании литературных данных и технологической оснащенности заводских лабораторий.

\section{Эксперимент}

В качестве объектов исследования фармацевтические субстанции, входящие в состав препаратов группы пенталгинов и их аналогов, обладающие противовоспалительным противопростудным и анальгезирующим действием. 
В работе использовали следующие методы. Спектры поглощения в ИКобласти получали на спектрометре ИК-Фурье «Avatar 360 FT-IR E.S.P» (Nicolet, США); Приставка однократного НПВО Smart Perfomer (оптический кристалл ZnSe): спектральный диапазон i.35+26.6 мкм, 7400-375 cм ${ }^{-1}$, предел допустимой абсолютной погрешности измерений по шкале волновых чисел $0.01 \mathrm{~cm}^{-1}$. Для ИКспектроскопии навески по 0.1 г двух веществ, помещали в агатовую ступку и тщательно растирали в течении 5 минут. Регистрировали спектры индивидуальных веществ (спектры снимались относительно воздуха) и ИК-спектр смеси, сравнивали его с суммарным спектром индивидуальных веществ. Смеси нагревали в сушильном шкафу в стеклянных бюксах, температура нагревания зависела от свойств соединений: смеси, в состав которых входят вещества с температурой плавления близкой к $100^{\circ} \mathrm{C}$ нагревали при температуре $90^{\circ} \mathrm{C}$, а остальные - при $100^{\circ} \mathrm{C}$. Bce смеси нагревали в течении 8 часов, периодически наблюдая изменения внешнего вида смесей.

Хроматограммы получали на ультраэффективном жидкостном хроматографе WATERS Acquity H-Class c диодно-матричным детектором PDA e $\lambda$ с рабочим

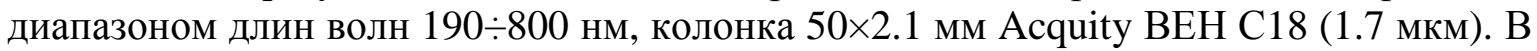
качестве буферной составляющей подвижной фазы применяли $0.4 \%$ раствор $\left(\mathrm{C}_{2} \mathrm{H}_{5}\right)_{3} \mathrm{~N}$ (добавление $\mathrm{CF}_{3} \mathrm{COOH}$ до $\mathrm{pH} 2.0$ ), а содержание ацетонитрила изменяли от 5 до 90 об. \% в течение 5 мин. Расход подвижной фазы составил $0.5 \mathrm{~cm}^{3} /$ мин, объем пробы 2 мкл. Детектирование вели при 263 нм. Изучаемые вещества и их модельные смеси подвергают обработке, максимально приближенной к технологическим условиям - смачиванию, совместному растиранию, сушке при повышенных температурах для исследовании нежелательных и (или) гипотетических взаимодействий изучаемые вещества. Изучение совместимости проводят в изотермических условиях: бинарные смеси выдерживают при $50^{\circ} \mathrm{C}$ и влажности 95\% в течение 12 дней, что [71] наиболее точно воспроизводит реальные условия хранения препарата. Для выявления и идентификации продуктов деструкции, а также для возможности обсуждения процессов, приводящих к деструкции, изучаемые соединения подвергли нагреванию в кислых $0.1 \mathrm{M} \mathrm{HC}$, и щелочных $0.1 \mathrm{M} \mathrm{NaOH}$, водных растворах, растворе пероксида водорода $3 \% \mathrm{H}_{2} \mathrm{O}_{2}$, порошки облучали УФ-светом и нагревали при температуре ниже точки плавления - $115^{\circ} \mathrm{C} 12$ часов. Полученные продукты подвергали хроматографированию в соответствии с представленными методиками.

Построение кривых охлаждения проведено на приборе Баумана-Фрома. Выбор калориметрического метода исследования процесса кристаллообразования с помощью кривых охлаждения обусловлен схожестью этого подхода с особенностями технологических режимов при производстве лекарственных препаратов. Образование твердой фазы из расплава характеризуется в интегральной форме термограммой в координатах $\mathrm{T}^{\circ} \mathrm{C}(\mathrm{t})$ с временным интервалом фиксации температуры в 10 с.

\section{Обсуждение результатов}

Для компонентов лекарственного препарата «Пенталгин Артро» (ОАО Фармстандарт-Лексредства, Россия) в модельных смесях показано наличие взаимодействия при совместном измельчении и нагревании. Взаимодействия выявлены в результате скринингового исследования методами ИК-спектроскопии и калориметрии, а также подтверждены ВЭЖХ анализом в результате выявления артефактов в модельных смесях. Применение ограниченного составом лекарствен-

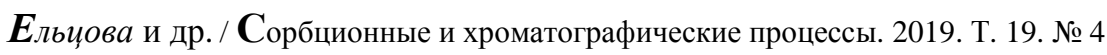


ной формы перечня фармацевтических субстанций не повлияло на обнаружение активных пар и результаты скринингового и рутинного исследований совпали.

С точки зрения теории, ИК спектры смесей должны отвечать правилу аддитивности, особенно в диапазонах активных функциональных групп. Поэтому любые отклонения от аддитивности были выявлены и исследованы дополнительно. Графический и цифровой анализ интенсивности поглощения проводили в узких спектральных диапазонах, на которых обнаружены различия суммарных спектров и спектров смеси. Проводили ранжирование отклонений интенсивностей поглощения по частотам и уточняли изменения в электронной структуре компонента. Выявлены отклонения от закона аддитивности ИК-спектров в парах компонентов лекарственного препарата «Пенталгин Артро» и других препаратов из группы жаропонижающих анальгетиков: дротаверин и магния, кальция стеарат, дротаверин и лимонная кислота, дротаверин и ГПЦ, дротаверин и натрия кроскармеллоза, напроксен и магния, кальция стеарат, напроксен и ГПЦ, напроксен и фенирамина малеат, фенирамина малеат и ГПЦ, фенирамина малеат и аскорбиновая кислота.

Для изучения термических свойств парных модельных смесей лекарственных субстанций по результатам эксперимента и расчетным данным строили диаграммы состояния, что было особенно информативно для смесей, взаимодействующих при совместном измельчении, например: аскорбиновая кислота - хлорфенирамин, аскорбиновая кислота - фенирамин, аскорбиновая кислота - фенилэфрин, напроксен фенирамина малеат.

Методом ВЭЖХ обнаружены продукты деструкции, что позволило определить тип процесса, приводящего к их образованию в растворах. Например, для напроксена и фенирамина малеата после воздействия стресс-факторов, а также в их смеси после нагревания показана вероятность деструктивных процессов окислительно-восстановительного характера. Исходя из спектров поглощения продуктов разложения возможно образование карбоксилатов малеиновой кислоты, поляризация третичной аминогруппы фенирамина. В смеси дротаверина со стеаратом магния выявлены продукты разложения (сумма 4.25\%) идентичные продуктам перекисного разложения дротаверина. В смесях дротаверина гидрохлорида и лимонной кислоты слабая кислота ведет себя как основание, что подтверждено наличием характерных продуктов (сумма 0,42\%). Смесь напроксена со стеаратом магния, несмотря на спектральные смещения, содержала незначительное количество примеси кислотного разложения. Незначительные количества выявленных продуктов деструкции не являются причиной не учитывать эти процессы при анализе готовой продукции.

Выводы ВЭЖХ анализа не полностью соответствовали результатам ИКспектроскопии и калориметрии. Это связано с влиянием растворителей и процессов хроматографического разделения.

По результатам опыта работы с группой многокомпонентных препаратов анальгетического, противовоспалительного и противопростудного действия сформулирован единый методический подход к процессу изучения стабильности фармацевтических субстанций в составе лекарственных композиций (рис.1).

- Выбор объектов может быть ограничен прописью лекарственного препарата или расширен за счет аналогов.

- Выявление отклонений в ИК-спектрах смеси и сумме спектров отдельных ингредиентов. Оценка термограмм и диаграмм состояния.

- Расчетно-графический анализ цифрового банка данных позволяет выявить продукты взаимодействия с большей уверенностью в результате.

- Метод ВЭЖХ (или его вариант УЭЖХ) обязательно используется для подтверждения отсутствия значимых количеств примесей и количественного содержа- 
ния активных компонентов и его применение на заключительном этапе позволяет доказать стабильность компонентов.

$\bullet$

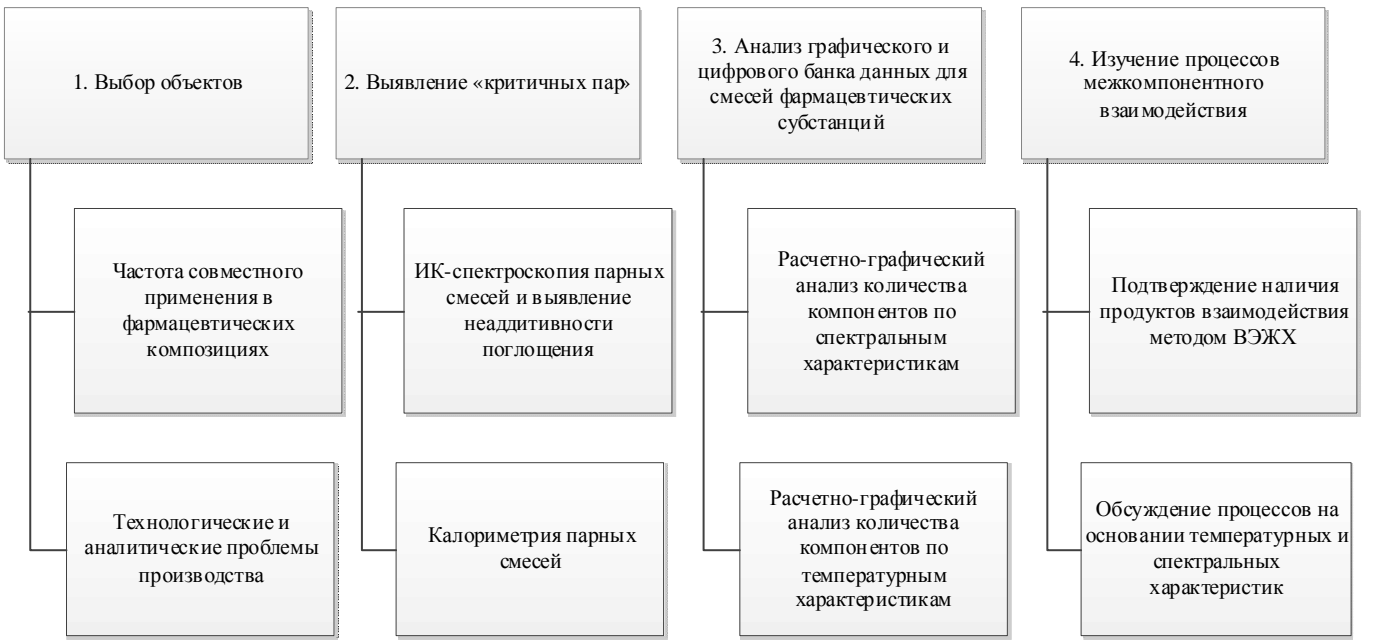

Рис. 1. Алгоритм изучения многокомпонентных фармацевтических смесей

\section{Заключение}

Актуальность и информативность ИК-спектроскопического, калориметрического и ВЭЖХ методов для анализа состояния многокомпонентных смесей фармацевтических субстанций входящих в состав лекарственных препаратов не подлежат сомнению. ИК-спектроскопия как метод качественного анализа включен во все современные фармакопеи. Калориметрические методы анализа являются классическими и актуальными для выявления взаимодействия веществ и определения их термодинамических параметров. При этом калориметрические и ИК-спектроскопические методы позволяют работать с системами без изменения фазового состояния. ВЭЖХ является рутинным методом анализа фармацевтических субстанций и лекарственных препаратов. Однако воздействие растворителей и неподвижных фаз способно изменять соотношение и состав компонентных систем, их нативную структуру, в частности наличие нековалентных связей в твердых композициях.

Изучение совместимости компонентов в системе обычно проводят в форме комплексных исследований. Обзор методов оценки стабильности показывает наибольшую частоту применения спектральных методов в сочетании с термическими (ДСК и ТГ) или хроматографическими (ВЭЖХ). При анализе литературы не выявлено исследований, объединяющих три метода: спектральные (в частности ИК-), хроматографические (ВЭЖХ) и исследование температур плавления. Комплексное применение оптических, хроматографических и термоаналитических методов в сочетании с воздействием стресс-факторов и (или) в условиях «ускоренного старения» позволило исследовать процессы и дать характеристику продуктов деструкции более эффективно, чем каждым из методов в отдельности. В результате проведенных исследований получены новые данные по стабильности 11 парных смесей: дротаверин и магния, кальция стеарат, дротаверин и лимонная кислота, дротаверин и ГПЦ, дротаверин и натрия кроскармеллоза, напроксен и магния, кальция стеарат, напроксен и ГПЦ, напроксен и фенирамина малеат, фенирамина малеат и ГПЦ, фенирамина малеат и аскорбиновая кислота, при совместном измельчении и нагревании которых усиливаются процессы межкомпонентного взаимодействия. 


\section{Список литературы}

1. Перцев И.М., Зупанц И.А. Фармацевтические и медико-биологические аспекты лекарств В 2 т. Харьков. УкраФа. 1999. С. 461.

2. Машковский М.Д.// Химикофармачевтический журн. 1995. Т. 29. № 7. C. 46-53.

3. Ельцова Н.О., Голубицкий Г.Б., Будко

Е.В. // Журнал аналитической химии. 2014. Т. 69. № 10. С. 1011-1023.

4. Гильдеева Г.Н., Чистяков В.В., Демченкова Е.Ю. // Химико-фармачевтический журнал. 2010. № 1. С. 43-45.

5. Tomar R.S., Joseph T.J., Murthy A.S.R. et al. // Journal of Pharmaceutical and Biomedical Analysis. 2004. Vol. 36. pp. 231-235.

\section{References}

1. Percev I.M., Zupanc I.A. Farmacevticheskie i mediko-biologicheskie aspekty lekarstv V 2 t. Har'kov: UkraFa. 1999. pp. 461.

2. Mashkovskij M.D., Khimikofarmacevticheskij zhurn, 1995, Vol. 29, No 7, pp. 46-53.

3. Eltsova N.O., Golubickij G.B., Budko E.V., Zhurnal analiticheskoj khimii, 2014, Vol. 69, No 10, pp. 1011-1023

4. Gil'deeva G.N., Chistjakov V.V., Demchenkova E.Yu., Khimiko-farmacevticheskij zhurnal, 2010, No 1, pp. 43-45.

5. Tomar R.S., Joseph T.J., Murthy A.S.R. et al., Journal of Pharmaceutical and Biomedical Analysis, 2004, Vol. 36, pp. 231-235.

Ельцова Наталья Олеговна - технолог, ООО «СТЦ» 195220, С.Петербург, тел. 89997215508

Будко Елена Вячеславовна - д.ф.н., професcop, зав. кафедры общей и биоорганической химии, Курский государственный медицинский университет, Курск
6. Pan C., Guan J., Lin M. // Journal of Pharmaceutical and Biomedical Analysis. 2011. Vol. 54. № 4. pp. 855-859.

7. Lin M., Li M., Buevich A.V. // Journal of Pharmaceutical and Biomedical Analysis. 2009. Vol. 50. pp. 275-280.

8. Raman B., Sharma B.A., Ghugare P.D. et al. // Journal of Pharmaceutical and Biomedical Analysis. 2009. Vol. 50. pp. 377-383.

9. Juhasz M., Kitahara Y., Takahashi S. et al. // Journal of Pharmaceutical and Biomedical Analysis. 2012. Vol. 59. pp. 190-193.

10. Ельцова Н.О., Будко Е.В., Ямпольский Л.М., Куликов А.Л. // Сорбционные и хроматографические прочессы. 2016. Т. 16. № 5. C. 719-723.

6. Pan C., Guan J., Lin M., Journal of Pharmaceutical and Biomedical Analysis, 2011, Vol. 54, No 4, pp. 855-859.

7. Lin M., Li M., Buevich A.V. et al., Journal of Pharmaceutical and Biomedical Analysis, 2009, Vol. 50, pp. 275-280.

8. Raman B., Sharma B.A., Ghugare P.D. et al., Journal of Pharmaceutical and Biomedical Analysis, 2009, Vol. 50, pp. 377-383.

9. Juhasz M., Kitahara Y., Takahashi S. et al., Journal of Pharmaceutical and Biomedical Analysis, 2012, Vol. 59, pp. 190-193.

10. Eltsova N.O., Budko E.V., Yampolskij L.M., Kulikov A.L., Sorbtsionnye i khromatograficheskie protsessy, 2016, Vol. 16, No 5, pp. 719-723.

Eltsova Natalia O. - technologist, LLC "STC" 195220, St. Petersburg St, e-mail: eltsova-n@mail.ru

Budko Elena V. - prof., grand Ph.D. (pharmacy), head of the department of general and bioorganic chemistry, Kursk state medical University, Kursk 